PROCEEDINGS OF THE

AMERICAN MATHEMATICAL SOCIETY

Volume 134, Number 5, Pages 1451-1457

S 0002-9939(05)08091-3

Article electronically published on October 18, 2005

\title{
INVERTIBILITY OF LINEAR COMBINATIONS OF TWO IDEMPOTENTS
}

\author{
HONGKE DU, XIYAN YAO, AND CHUNYUAN DENG
}

(Communicated by Joseph A. Ball)

\begin{abstract}
Let $P$ and $Q$ be two idempotents on a Hilbert space. In this note, we prove that the invertibility of the linear combination $\lambda_{1} P+\lambda_{2} Q$ is independent of the choice of $\lambda_{i}, i=1,2$, if $\lambda_{1} \lambda_{2} \neq 0$ and $\lambda_{1}+\lambda_{2} \neq 0$.
\end{abstract}

Let $\mathcal{H}$ be a Hilbert space, and let all bounded linear operators on $\mathcal{H}$ be denoted by $\mathcal{B}(\mathcal{H})$. An operator $P \in \mathcal{B}(\mathcal{H})$ is said to be idempotent if $P^{2}=P$. The set $\mathcal{P}$ of all idempotents in $\mathcal{B}(\mathcal{H})$ is invariant under similarity; that is, if $P \in \mathcal{P}$ and $S \in \mathcal{B}(\mathcal{H})$ is an invertible operator, then $S^{-1} P S$ is still an idempotent since $\left(S^{-1} P S\right)^{2}=S^{-1} P S S^{-1} P S=S^{-1} P^{2} S=S^{-1} P S$. An idempotent $P$ is called an orthogonal projection if $P^{2}=P=P^{*}$, where $P^{*}$ is the adjoint of $P$. Moreover, for an idempotent $P \in \mathcal{P}$, there exists an invertible operator $U \in \mathcal{B}(\mathcal{H})$ such that $U^{-1} P U$ is an orthogonal projection. In fact, if $P \in \mathcal{P}$, then $P$ can be written in the form of

$$
P=\left(\begin{array}{cc}
I & P_{1} \\
0 & 0
\end{array}\right)
$$

with respect to the space decomposition $\mathcal{H}=\mathcal{R}(P) \oplus \mathcal{R}(P)^{\perp}$, where $\mathcal{R}(M)$ denotes the range of the operator $M$. In this case, we have

$$
\left(\begin{array}{cc}
I & P_{1} \\
0 & I
\end{array}\right)\left(\begin{array}{cc}
I & P_{1} \\
0 & 0
\end{array}\right)\left(\begin{array}{cc}
I & -P_{1} \\
0 & I
\end{array}\right)=\left(\begin{array}{ll}
I & 0 \\
0 & 0
\end{array}\right),
$$

where $\tilde{P}=\left(\begin{array}{cc}I & -P_{1} \\ 0 & I\end{array}\right)$ is invertible and $\tilde{P}^{-1}=\left(\begin{array}{cc}I & P_{1} \\ 0 & I\end{array}\right)$. An operator $A \in$ $\mathcal{B}(\mathcal{H})$ is said to be positive if $(A x, x) \geq 0$ for all $x \in \mathcal{H}$. If $A$ is positive, then $A^{\frac{1}{2}}$ denotes the positive square root of $A$.

In recent years, a number of researchers have considered questions concerning the idempotents and linear combinations of idempotents (see [1]- 8 ). Particularly, some researchers pay much attention to the study of linear combinations of two idempotents ([1, [5]). For example, if $P_{i}, i=1,2$, are idempotents in the finitedimensional space $C^{n}$, J. K. Baksalary and O. M. Baksalary ([1]) have proved that the nonsingularity of $P_{1}+P_{2}$ is equivalent to the nonsingularity of any linear combination $c_{1} P_{1}+c_{2} P_{2}$, where $c_{1}+c_{2} \neq 0$. In the present note, we will study the

Received by the editors June 19, 2004 and, in revised form, December 20, 2004.

2000 Mathematics Subject Classification. Primary 47A05, 47L07.

Key words and phrases. Idempotent, invertibility, linear combination of two idempotents.

This research was partially supported by the National Natural Science Foundation of China (19771056).

(C)2005 American Mathematical Society Reverts to public domain 28 years from publication 
invertibility of linear combinations of two idempotents on an infinite-dimensional Hilbert space. We obtain the main result, which is similar to [1, but the idea of the proof is different from 1 .

Theorem 1. Let $P$ and $Q$ in $\mathcal{B}(\mathcal{H})$ be two idempotents. If $\lambda_{1}$ and $\lambda_{2}$ are nonzero complex numbers and $\lambda_{1}+\lambda_{2} \neq 0$, then the invertibility of $\lambda_{1} P+\lambda_{2} Q$ is independent of the choice of $\lambda_{i}, i=1,2$.

To prove Theorem 1, we need some lemmas which are well known, so the proofs are omitted.

Lemma 2. Let $A=\left(\begin{array}{ll}A_{11} & A_{12} \\ A_{21} & A_{22}\end{array}\right)$ be a bounded linear operator on $\mathcal{H} \oplus \mathcal{K}$. Then $A$ is a positive operator if and only if $A_{11} \geq 0, A_{22} \geq 0, A_{12}=A_{21}^{*}$ and there exists a contraction $D$ from $\mathcal{K}$ into $\mathcal{H}$ such that

$$
A=\left(\begin{array}{cc}
A_{11} & A_{11}^{\frac{1}{2}} D A_{22}^{\frac{1}{2}} \\
A_{22}^{\frac{1}{2}} D^{*} A_{11}^{\frac{1}{2}} & A_{22}
\end{array}\right) .
$$

Lemma 3. Let $A \in \mathcal{B}(\mathcal{H})$ be invertible and $\widetilde{A}=\left(\begin{array}{ll}A & B \\ C & D\end{array}\right) \in \mathcal{B}(\mathcal{H} \oplus \mathcal{K})$. Then $\widetilde{A}$ is invertible if and only if $D-C A^{-1} B$ is invertible.

Lemma 4. Let $A \in \mathcal{B}(\mathcal{H})$ be a positive operator. Then the following statements hold:

(1) $\mathcal{R}(A) \subseteq \mathcal{R}\left(A^{\frac{1}{2}}\right)$ and $\overline{\mathcal{R}(A)}=\overline{\mathcal{R}\left(A^{\frac{1}{2}}\right)}$, where $\bar{K}$ denotes the closure of $K$;

(2) $\mathcal{R}(A)$ is closed if and only if $\mathcal{R}(A)=\mathcal{R}\left(A^{\frac{1}{2}}\right)$;

(3) $\mathcal{R}(A)=\mathcal{H}$ if and only if $A$ is invertible.

Proof. Let $P$ and $Q$ be two idempotents. By the discussion above, since $\lambda_{1} P+$ $\lambda_{2} Q$ is invertible if and only if $\lambda_{1} S^{-1} P S+\lambda_{2} S^{-1} Q S$ is invertible, to consider the invertibility of $\lambda_{1} P+\lambda_{2} Q$, without loss of generality, we can assume that one of $P$ and $Q$ is an orthogonal projection. For example, assume that $Q$ is an orthogonal projection. Of course, $Q$ is a positive operator. In this case, by Lemma $2, P$ and $Q$ have the following operator matrix forms:

$$
P=\left(\begin{array}{cc}
I & P_{1} \\
0 & 0
\end{array}\right) \text { and } Q=\left(\begin{array}{cc}
Q_{1} & Q_{1}^{\frac{1}{2}} D Q_{2}^{\frac{1}{2}} \\
Q_{2}^{\frac{1}{2}} D^{*} Q_{1}^{\frac{1}{2}} & Q_{2}
\end{array}\right)
$$

with respect to the space decomposition $\mathcal{H}=\mathcal{R}(P) \oplus \mathcal{R}(P)^{\perp}$, where $Q_{1}$ and $Q_{2}$ are positive operators on $\mathcal{R}(P)$ and $\mathcal{R}(P)^{\perp}$, respectively, and $D$ is a contraction operator from $\mathcal{R}(P)^{\perp}$ into $\mathcal{R}(P)$.

Suppose $\lambda_{1} \neq 0$ and $\lambda_{2} \neq 0$. If $\lambda_{1} P+\lambda_{2} Q$ is invertible, that is, the operator matrix

$$
\lambda_{1} P+\lambda_{2} Q=\left(\begin{array}{cc}
\lambda_{1} I+\lambda_{2} Q_{1} & \lambda_{1} P_{1}+\lambda_{2} Q_{1}^{\frac{1}{2}} D Q_{2}^{\frac{1}{2}} \\
\lambda_{2} Q_{2}^{\frac{1}{2}} D^{*} Q_{1}^{\frac{1}{2}} & \lambda_{2} Q_{2}
\end{array}\right)
$$

is invertible, then $\mathcal{R}\left(\left(\lambda_{2} Q_{2}^{\frac{1}{2}} D^{*} Q_{1}^{\frac{1}{2}}, \lambda_{2} Q_{2}\right)\right)=\mathcal{R}(P)^{\perp}$. By Lemma $4, \mathcal{R}\left(Q_{2}\right) \subseteq$ $\mathcal{R}\left(Q_{2}^{\frac{1}{2}}\right)$ and observing that $\mathcal{R}\left(\left(\lambda_{2} Q_{2}^{\frac{1}{2}} D^{*} Q_{1}^{\frac{1}{2}}, \lambda_{2} Q_{2}\right)\right) \subseteq \mathcal{R}\left(Q_{2}\right)^{\frac{1}{2}} \subseteq \mathcal{R}(P)^{\perp}$, then

$$
\mathcal{R}\left(Q_{2}^{\frac{1}{2}}\right)=\mathcal{R}(P)^{\perp} \text {. }
$$

By Lemma 4 again we have

$$
\mathcal{R}\left(Q_{2}\right)=\mathcal{R}(P)^{\perp}
$$


This shows that $Q_{2}$ is invertible. In this case, by Lemma $3, \lambda_{1} P+\lambda_{2} Q$ is invertible if and only if

is invertible.

$$
\lambda_{1} I+\lambda_{2} Q_{1}-\left(\lambda_{1} P_{1} Q_{2}^{-\frac{1}{2}}+\lambda_{2} Q_{1}^{\frac{1}{2}} D\right) D^{*} Q_{1}^{\frac{1}{2}}
$$

Since $Q_{1}$ is a positive contraction on $\mathcal{R}(P)$ and $Q_{2}$ is an invertible positive contraction on $\mathcal{R}(P)^{\perp}$, then $Q_{1}$ as an operator on $\mathcal{R}(P)$ and $Q_{2}$ as an operator on $\mathcal{R}(P)^{\perp}$ have the following operator matrix forms:

$$
Q_{1}=\left(\begin{array}{ccc}
0 & 0 & 0 \\
0 & I & 0 \\
0 & 0 & Q_{11}
\end{array}\right), Q_{2}=\left(\begin{array}{cc}
Q_{22} & 0 \\
0 & I
\end{array}\right)
$$

with respect to the space decomposition

$$
\mathcal{R}(P)=\mathcal{N}\left(Q_{1}\right) \oplus \mathcal{N}\left(I-Q_{1}\right) \oplus\left(\mathcal{R}(P) \ominus\left(\mathcal{N}\left(Q_{1}\right) \oplus \mathcal{N}\left(I-Q_{1}\right)\right)\right)
$$

and the space decomposition

$$
\mathcal{R}(P)^{\perp}=\left(\mathcal{R}(P)^{\perp} \ominus \mathcal{N}\left(I-Q_{2}\right)\right) \oplus \mathcal{N}\left(I-Q_{2}\right),
$$

respectively.

Then denote $\mathcal{H}_{0}=\mathcal{N}\left(Q_{1}\right), \mathcal{H}_{1}=\mathcal{N}\left(I-Q_{1}\right), \mathcal{H}_{2}=\mathcal{R}(P) \ominus\left(\mathcal{N}\left(Q_{1}\right) \oplus \mathcal{N}\left(I-Q_{1}\right)\right)$, $\mathcal{H}_{3}=\mathcal{R}(P)^{\perp} \ominus \mathcal{N}\left(I-Q_{2}\right)$ and $\mathcal{H}_{4}=\mathcal{N}\left(I-Q_{2}\right), Q_{11}$ and $I-Q_{11}$ are injective positive contractions on $\mathcal{H}_{2}$ and $Q_{22}$ is an invertible positive contraction on $\mathcal{H}_{3}$ with $1 \notin \sigma_{p}\left(Q_{22}\right)$, where $\sigma_{p}(M)$ denotes the point spectrum of the operator $M$. In this case, $P$ and $Q$ have the following matrix representations:

$$
Q=\left(\begin{array}{ccccc}
0 & 0 & 0 & 0 & 0 \\
0 & I & 0 & 0 & 0 \\
0 & 0 & Q_{11} & Q_{11}^{\frac{1}{2}} D_{1} Q_{22}^{\frac{1}{2}} & 0 \\
0 & 0 & Q_{22}^{\frac{1}{2}} D_{1}^{*} Q_{11}^{\frac{1}{2}} & Q_{22} & 0 \\
0 & 0 & 0 & 0 & I
\end{array}\right)
$$

and

$$
P=\left(\begin{array}{ccccc}
I & 0 & 0 & P_{11} & P_{12} \\
0 & I & 0 & P_{21} & P_{22} \\
0 & 0 & I & P_{31} & P_{32} \\
0 & 0 & 0 & 0 & 0 \\
0 & 0 & 0 & 0 & 0
\end{array}\right)
$$

with respect to the space decomposition $\mathcal{H}=\bigoplus_{i=0}^{4} \mathcal{H}_{i}$. If we let

$$
Q_{0}=\left(\begin{array}{cc}
Q_{11} & Q_{11}^{\frac{1}{2}} D_{1} Q_{22}^{\frac{1}{2}} \\
Q_{22}^{\frac{1}{2}} D_{1}^{*} Q_{11}^{\frac{1}{2}} & Q_{22}
\end{array}\right),
$$

then $Q$ being an orthogonal projection implies that $Q_{0}$ is also an orthogonal projection on $\mathcal{H}_{2} \oplus \mathcal{H}_{3}$. That is, $Q_{0}=Q_{0}^{2}$. Hence we have

$$
\begin{aligned}
Q_{0}^{2} & =\left(\begin{array}{cc}
Q_{11} & Q_{11}^{\frac{1}{2}} D_{1} Q_{22}^{\frac{1}{2}} \\
Q_{22}^{\frac{1}{2}} D_{1}^{*} Q_{11}^{\frac{1}{2}} & Q_{22}
\end{array}\right)\left(\begin{array}{cc}
Q_{11} & Q_{11}^{\frac{1}{2}} D_{1} Q_{22}^{\frac{1}{2}} \\
Q_{22}^{\frac{1}{2}} D_{1}^{*} Q_{11}^{\frac{1}{2}} & Q_{22}
\end{array}\right) \\
& =\left(\begin{array}{cc}
Q_{11}^{2}+Q_{11}^{\frac{1}{2}} D_{1} Q_{22} D_{1}^{*} Q_{11}^{\frac{1}{2}} & Q_{11}^{\frac{3}{2}} D_{1} Q_{22}^{\frac{1}{2}}+Q_{11}^{\frac{1}{2}} D_{1} Q_{22}^{\frac{3}{2}} \\
Q_{22}^{\frac{3}{2}} D_{1}^{*} Q_{11}^{\frac{1}{2}}+Q_{22}^{\frac{1}{2}} D_{1}^{*} Q_{11}^{\frac{3}{2}} & Q_{22}^{2}+Q_{22}^{\frac{1}{2}} D_{1}^{*} Q_{11} D_{1} Q_{22}^{\frac{1}{2}}
\end{array}\right)
\end{aligned}
$$


and

$$
\begin{aligned}
& \left(\begin{array}{cc}
Q_{11} & Q_{11}^{\frac{1}{2}} D_{1} Q_{22}^{\frac{1}{2}} \\
Q_{22}^{\frac{1}{2}} D_{1}^{*} Q_{11}^{\frac{1}{2}} & Q_{22}
\end{array}\right) \\
& =\left(\begin{array}{cc}
Q_{11}^{2}+Q_{11}^{\frac{1}{2}} D_{1} Q_{22} D_{1}^{*} Q_{11}^{\frac{1}{2}} & Q_{11}^{\frac{3}{2}} D_{1} Q_{22}^{\frac{1}{2}}+Q_{11}^{\frac{1}{2}} D_{1} Q_{22}^{\frac{3}{2}} \\
Q_{22}^{\frac{3}{2}} D_{1}^{*} Q_{11}^{\frac{1}{2}}+Q_{22}^{\frac{1}{2}} D_{1}^{*} Q_{11}^{\frac{3}{2}} & Q_{22}^{2}+Q_{22}^{\frac{1}{2}} D_{1}^{*} Q_{11} D_{1} Q_{22}^{\frac{1}{2}}
\end{array}\right) .
\end{aligned}
$$

Comparing the two sides of the above equation, we have

$$
\left\{\begin{array}{l}
Q_{11}=Q_{11}^{2}+Q_{11}^{\frac{1}{2}} D_{1} Q_{22} D_{1}^{*} Q_{11}^{\frac{1}{2}}, \\
Q_{11}^{\frac{1}{2}} D_{1} Q_{22}^{\frac{1}{2}}=Q_{11}^{\frac{3}{2}} D_{1} Q_{22}^{\frac{1}{2}}+Q_{11}^{\frac{1}{2}} D_{1} Q_{22}^{\frac{3}{2}}, \\
Q_{22}^{\frac{1}{2}} D_{1}^{*} Q_{11}^{\frac{1}{2}}=Q_{22}^{\frac{3}{2}} D_{1}^{*} Q_{11}^{\frac{1}{2}}+Q_{22}^{\frac{1}{2}} D_{1}^{*} Q_{11}^{\frac{3}{2}}, \\
Q_{22}=Q_{22}^{2}+Q_{22}^{\frac{1}{2}} D_{1}^{*} Q_{11} D_{1} Q_{22}^{\frac{1}{2}} .
\end{array}\right.
$$

Observing that $Q_{11}, I_{\mathcal{H}_{2}}-Q_{11}, Q_{22}$ and $I_{\mathcal{H}_{3}}-Q_{22}$ are injective, we get

$$
\left\{\begin{array}{l}
I_{\mathcal{H}_{2}}=Q_{11}+D_{1} Q_{22} D_{1}^{*} \\
D_{1}=Q_{11} D_{1}+D_{1} Q_{22} \\
D_{1}^{*}=Q_{22} D_{1}^{*}+D_{1}^{*} Q_{11} \\
I_{\mathcal{H}_{3}}=Q_{22}+D_{1}^{*} Q_{11} D_{1}
\end{array}\right.
$$

where $I_{\mathcal{H}_{i}}$ denotes the identity on $\mathcal{H}_{i}, i=2,3$. From the last of equations (3), we see that $I_{\mathcal{H}_{3}}-Q_{22}=D_{1}^{*} Q_{11} D_{1}$, and hence $Q_{11} D_{1}$ is injective. From the second of equations (3) we see that

$$
\left(I_{\mathcal{H}_{2}}-Q_{11}\right) D_{1}=D_{1} Q_{22} .
$$

On the other hand, from the first of equations (3) we get

$$
\left(I_{\mathcal{H}_{2}}-Q_{11}\right) D_{1}=D_{1} Q_{22} D_{1}^{*} D_{1} .
$$

Equating these two expressions for $\left(I_{\mathcal{H}_{2}}-Q_{11}\right) D_{1}$ and using the injectivity of $D_{1} Q_{22}$ then leaves us with

$$
I_{\mathcal{H}_{3}}=D_{1}^{*} D_{1} \text {. }
$$

In a similar vein, from the second of equations (3) we have $D_{1}\left(I_{\mathcal{H}_{3}}-Q_{22}\right)=Q_{11} D_{1}$, while from the last of equations (3) we have $D_{1}\left(I_{\mathcal{H}_{3}}-Q_{22}\right)=D_{1} D_{1}^{*} Q_{11} D_{1}$. Equating these two expressions for $D_{1}\left(I_{\mathcal{H}_{3}}-Q_{22}\right)$ and using the injectivity of $Q_{11} D_{1}$ then gives

$$
I_{\mathcal{H}_{2}}=D_{1} D_{1}^{*}
$$

With this identity, the first of equations (3) can then be rewritten as

$$
Q_{11}=D_{1}\left(I_{\mathcal{H}_{3}}-Q_{22}\right) D_{1}^{*}
$$

We have thus arrived at the system of equations

$$
\left\{\begin{array}{l}
D_{1} D_{1}^{*}=I_{\mathcal{H}_{2}}, \\
D_{1}^{*} D_{1}=I_{\mathcal{H}_{3}}, \\
Q_{11}=D_{1}\left(I_{\mathcal{H}_{3}}-Q_{22}\right) D_{1}^{*}
\end{array}\right.
$$

Denote $\hat{Q}=Q_{11}$. Then

$$
Q_{0}=\left(\begin{array}{cc}
\hat{Q} & \hat{Q}^{\frac{1}{2}}(I-\hat{Q})^{\frac{1}{2}} D_{1} \\
D_{1}^{*} \hat{Q}^{\frac{1}{2}}(I-\hat{Q})^{\frac{1}{2}} & D_{1}^{*}(I-\hat{Q}) D_{1}
\end{array}\right) .
$$


Now $\lambda_{1} P+\lambda_{2} Q$ has the following operator matrix form:

$$
\begin{aligned}
& \lambda_{1} P+\lambda_{2} Q=\lambda_{1}\left(\begin{array}{ccccc}
I & 0 & 0 & P_{11} & P_{12} \\
0 & I & 0 & P_{21} & P_{22} \\
0 & 0 & I & P_{31} & P_{32} \\
0 & 0 & 0 & 0 & 0 \\
0 & 0 & 0 & 0 & 0
\end{array}\right) \\
& +\lambda_{2}\left(\begin{array}{ccccc}
0 & 0 & 0 & 0 & 0 \\
0 & I & 0 & 0 & 0 \\
0 & 0 & Q_{11} & Q_{11}^{\frac{1}{2}} D_{1} Q_{22}^{\frac{1}{2}} & 0 \\
0 & 0 & Q_{22}^{\frac{1}{2}} D_{1}^{*} Q_{11}^{\frac{1}{2}} & Q_{22} & 0 \\
0 & 0 & 0 & 0 & I
\end{array}\right) \\
& =\left(\begin{array}{ccccc}
\lambda_{1} I & 0 & 0 & \lambda_{1} P_{11} & \lambda_{1} P_{12} \\
0 & \left(\lambda_{1}+\lambda_{2}\right) I & 0 & \lambda_{1} P_{21} & \lambda_{1} P_{22} \\
0 & 0 & \lambda_{1} I+\lambda_{2} \hat{Q} & \lambda_{1} P_{31}+\lambda_{2} \hat{Q}^{\frac{1}{2}}(I-\hat{Q})^{\frac{1}{2}} D_{1} & \lambda_{1} P_{32} \\
0 & 0 & \lambda_{2} D_{1}^{*} \hat{Q}^{\frac{1}{2}}(I-\hat{Q})^{\frac{1}{2}} & \lambda_{2} D_{1}^{*}(I-\hat{Q})^{\frac{1}{2}} D_{1} & 0 \\
0 & 0 & 0 & 0 & I
\end{array}\right)
\end{aligned}
$$

with respect to the space decomposition $\mathcal{H}=\bigoplus_{i=0}^{4} \mathcal{H}_{i}$.

Denote

$$
\bar{Q}_{0}=\left(\begin{array}{cc}
\lambda_{1} I+\lambda_{2} \hat{Q} & \lambda_{1} P_{31}+\lambda_{2} \hat{Q}^{\frac{1}{2}}(I-\hat{Q})^{\frac{1}{2}} D_{1} \\
\lambda_{2} D_{1}^{*} \hat{Q}^{\frac{1}{2}}(I-\hat{Q})^{\frac{1}{2}} & \lambda_{2} D_{1}^{*}(I-\hat{Q}) D_{1}
\end{array}\right) .
$$

Obviously, if $\lambda_{1}+\lambda_{2} \neq 0$, the invertibility of the operator $\lambda_{1} P+\lambda_{2} Q$ on $\mathcal{H}$ is equivalent to the invertibility of $\bar{Q}_{0}$ on $\mathcal{H}_{2} \oplus \mathcal{H}_{3}$. Moreover, by Lemma 3 , that $\bar{Q}_{0}$ is invertible if and only if $I-\hat{Q}$ and

$$
\lambda_{1} I+\lambda_{2} \hat{Q}-\left(\lambda_{1} P_{31}+\lambda_{2} \hat{Q}^{\frac{1}{2}}(I-\hat{Q})^{\frac{1}{2}} D_{1}\right) \lambda_{2}^{-1} D_{1}^{*}(I-\hat{Q})^{-1} D_{1} \lambda_{2} D_{1}^{*} \hat{Q}^{\frac{1}{2}}(I-\hat{Q})^{\frac{1}{2}}
$$

are invertible. Noting that $D_{1} D_{1}^{*}=I_{\mathcal{H}_{2}}$ and $\lambda_{1} \lambda_{2} \neq 0$, we have

$$
\begin{aligned}
& \lambda_{1} I+\lambda_{2} \hat{Q}-\left(\lambda_{1} P_{31}+\lambda_{2} \hat{Q}^{\frac{1}{2}}(I-\hat{Q})^{\frac{1}{2}} D_{1}\right) \lambda_{2}^{-1} D_{1}^{*}(I-\hat{Q})^{-1} D_{1} \lambda_{2} D_{1}^{*} \hat{Q}^{\frac{1}{2}}(I-\hat{Q})^{\frac{1}{2}} \\
& =\lambda_{1}\left(I-P_{31} D_{1}^{*} \hat{Q}^{\frac{1}{2}}(I-\hat{Q})^{-\frac{1}{2}}\right) .
\end{aligned}
$$

This shows that the invertibility of $\bar{Q}_{0}$ is only dependent on the invertibility of the operator $I-P_{31} D_{1}^{*} \hat{Q}^{\frac{1}{2}}(I-\hat{Q})^{-\frac{1}{2}}$ if both $\lambda_{1}$ and $\lambda_{2}$ are not zero; that is, the invertibility of $\bar{Q}_{0}$ is independent of the choice of $\lambda_{1}$ and $\lambda_{2}$ if both $\lambda_{1}$ and $\lambda_{2}$ are not zero.

In other words, the invertibility of $\lambda_{1} P+\lambda_{2} Q$ is independent of the choice of $\lambda_{1}$ and $\lambda_{2}$ if both $\lambda_{1}$ and $\lambda_{2}$ are not zero and $\lambda_{1}+\lambda_{2} \neq 0$.

Remark. By the proof of Theorem 1, if $\mathcal{R}(P) \cap \mathcal{R}(Q)=\{0\}$, the invertibility of $\lambda_{1} P+\lambda_{2} Q$ is independent of the choice of $\lambda_{1}$ and $\lambda_{2}$ if both $\lambda_{1}$ and $\lambda_{2}$ are not zero.

The following consequence is immediate.

Corollary 5. Let $P$ and $Q$ be two idempotents and $\mathcal{R}(P) \cap \mathcal{R}(Q)=\{0\}$. Then the following statements hold:

(1) $P+Q$ is invertible if and only if $P-Q$ is invertible;

(2) In addition, $\mathcal{R}(\tilde{P}) \cap \mathcal{R}(\tilde{Q})=\{0\}$, and $P+Q$ is invertible if and only if $\tilde{P}+\tilde{Q}$ is invertible, where $\tilde{K}=I-K$. 
Proof. (1) It is clear from the Remark above.

(2) Obviously, $\tilde{P}$ and $\tilde{Q}$ are idempotents. Since $\mathcal{R}(\tilde{P}) \cap \mathcal{R}(\tilde{Q})=\{0\}, \tilde{P}+\tilde{Q}$ is invertible if and only if $\tilde{P}-\tilde{Q}$ is invertible by (1). But

$$
\tilde{P}-\tilde{Q}=I-P-(I-Q)=-(P-Q),
$$

so $\tilde{P}-\tilde{Q}$ is invertible if and only if $P-Q$ is invertible. By (1), $P-Q$ is invertible if and only if $P+Q$ is invertible.

Corollary 6. Let $P$ and $Q$ be two orthogonal projections such that $P+Q$ is invertible and $\mathcal{R}(P) \cap \mathcal{R}(Q)=\{0\}$. Then $P-Q, 1-P Q, P+Q-P Q$ are all invertible.

Proof. If $\mathcal{R}(P) \cap \mathcal{R}(Q)=\{0\}$, then $\mathcal{H}_{1}=\{0\}$. Observe that in the proof of Theorem $1, P+Q$ has the following operator matrix:

$$
\begin{aligned}
P+Q & =\left(\begin{array}{cccc}
I & 0 & 0 & 0 \\
0 & I & 0 & 0 \\
0 & 0 & 0 & 0 \\
0 & 0 & 0 & 0
\end{array}\right)+\left(\begin{array}{cccc}
0 & 0 & 0 & 0 \\
0 & Q_{11} & Q_{11}^{\frac{1}{2}} D_{1} Q_{22}^{\frac{1}{2}} & 0 \\
0 & Q_{22}^{\frac{1}{2}} D_{1}^{*} Q_{11}^{\frac{1}{2}} & Q_{22} & 0 \\
0 & 0 & 0 & I
\end{array}\right) \\
& =\left(\begin{array}{cccc}
I & 0 & 0 & 0 \\
0 & I+Q_{11} & Q_{11}^{\frac{1}{2}}\left(I-Q_{11}\right)^{\frac{1}{2}} D_{1} & 0 \\
0 & D_{1}^{*} Q_{11}^{\frac{1}{2}}\left(I-Q_{11}\right)^{\frac{1}{2}} & D_{1}^{*}\left(I-Q_{11}\right)^{\frac{1}{2}} D_{1} & 0 \\
0 & 0 & 0 & I
\end{array}\right)
\end{aligned}
$$

with respect to the space decomposition $\mathcal{H}=\mathcal{H}_{0} \oplus\left(\bigoplus_{i=2}^{4} \mathcal{H}_{i}\right)$. Then the invertibility of $P+Q$ implies that $I-Q_{11}$ is invertible. A direct calculation can show that $1-P Q$ and $P+Q-P Q$ are all invertible.

\section{ACKNOWLEDGMENT}

A part of this paper was written while the first author visited the Department of Mathematics and Statistics of the University of New Hampshire during the summer of 2004. He would like to thank the faculty and administration of those units for their warm hospitality. In particular, the first author wants to express his gratitude to Professor Liming Ge for his useful suggestions and comments.

The authors wish to thank the referees for their valuable comments and suggestions.

\section{REFERENCES}

[1] J. K. Baksalary and O. M. Baksalary, Nonsingularity of linear combinations of idempotent matrices, Linear Algebra and its Applications, Volume 388, 1 September 2004, 25-29. MR.2077846

[2] O. M. Baksalary, Idempotency of linear combinations of three idempotent matrices, two of which are disjoint, Linear Algebra and its Applications, Volume 388 (2004), 67-78. MR2077851

[3] Man-Duen Choi and Pei Yuan Wu, Convex combinations of projections, Linear Algebra and its Applications, Volume 136, 15 July (1990), 25-42. MR1061537 (91e:15018)

[4] G. Corach, A. Maestripieri and D. Stojanoff, Generalized Schur complements and oblique projections, Linear Algebra and its Applications, Volume 341, Issues 1-3, 15 January (2002), 259-272. MR 1873624 (2003b:47035)

[5] J. Gross and G. Trenkler, Nonsingularity of the Difference of Two Oblique Projectors. SIAM Journal on Matrix Analysis and Applications, Volume 21, Number 2 (1999), 390-395. MR:1718336 (2000h:15003) 
[6] S. Kruglyak, V. Rabanovich and Y. Samolenko, Decomposition of a scalar matrix into a sum of orthogonal projections, Linear Algebra and its Applications, Volume 370, 1 September (2003), 217-225. MR1994329 (2004f:15045)

[7] J. J. Koliha, V. Rakoevi and I. Strakraba, The difference and sum of projectors, Linear Algebra and its Applications, Volume 388, 1 September 2004, 279-288. MR2077865

[8] E. Spiegel, Sums of projections, Linear Algebra and its Applications, Volume 187, 1 July 1993, Pages 239-249. MR1221707 (94m:15001)

College of Mathematics and Information Science, ShaAnxi Normal University, Xi'an 710062, People's Republic of China

E-mail address: hkdu@snnu.edu.cn

College of Mathematics and Information Science, Shaanxi Normal University, Xi'an 710062, People's Republic of China

E-mail address: yaoxiyan63@163.com

College of Mathematics and Information Science, ShaAnXi Normal University, Xi'an 710062, People's Republic of China

E-mail address: cy-deng@263.net 